\title{
Tendances en matière de cancer au Canada, 1984-2015
}

\author{
Alain A. Demers, Ph. D. (1,2); Darren R. Brenner, Ph. D. (3); Leah Smith, Ph. D. (4); Amanda Shaw, M. Sc. (1)
}

Diffuser cet article sur Twitter

\section{Résumé}

L'étude des tendances portant sur l'incidence de tous les types de cancers confondus effectuée afin de comprendre les tendances en matière de cancer peut donner lieu à des conclusions erronées, car ces tendances sont variables selon le type de cancer. Cet article présente les résultats concernant les tendances tirées des Statistiques canadiennes sur le cancer 2019 en utilisant la variation annuelle en pourcentage (VAP) des taux d'incidence normalisés selon l'âge. Parmi les résultats, notons une hausse récente du taux d'incidence du cancer de la glande thyroïde chez les hommes (VAP : 6,4 \%, 1997-2015) ainsi qu'une diminution des taux d'incidence du cancer de la prostate (VAP : $-9,1 \%, 2011-2015$ ) et du cancer du col de l'utérus (VAP : - 3,3 \%, 2010-2015).

Mots-clés : néoplasmes, analyse des données, tendance, cancer

\section{Introduction}

On considère souvent que les tendances en matière de maladies chroniques sont stables ou offrent de faibles variations au sein des populations. Par exemple, le taux d'incidence du diabète au Canada est passé de 6,7 pour 1000 habitants en 2003-2004 à 6,3 pour 1000 habitants en 2013-2014¹. Jusqu'en 2011, avant que les taux d'incidence de cancer de la prostate amorcent un déclin rapide, cette perception de stabilité relative pouvait également s'appliquer à l'ensemble des cancers dans la mesure où ils étaient présentés de façon groupée. Selon le rapport Statistiques canadiennes sur le cancer 2019 (SCC 2019), entre 1984 et 2015, les taux d'incidence du cancer au Canada ont ainsi augmenté de 0,1\% par année en moyenne ${ }^{2}$. Cependant, ce portrait collectif est trompeur, car les tendances ont varié de manière considérable selon le type de cancer et la période ${ }^{2}$.

La surveillance de l'incidence de chaque type de cancer au fil du temps peut permettre de relever les tendances émergentes et de mettre en évidence les secteurs où des progrès ont été réalisés et ceux où des ressources et des travaux additionnels sont nécessaires. Cet article a pour objet de présenter les résultats du rapport SCC 2019 en matière d'évolution des tendances liées à l'incidence des cancers au Canada, en accordant une attention particulière aux profils de variation pour chaque type de cancer.

\section{Méthodologie}

Les données sont tirées du chapitre sur l'incidence des cancers du rapport SCC 2019², qui porte sur la période 1984 à 2015. Le Québec n’a pas été inclus dans ce rapport, car les données pour cette province n'étaient disponibles que jusqu'en 2010. Le Registre canadien du cancer (RCC) ${ }^{3}$ a été utilisé comme source de données pour la période 1992 à 2015 et le Système national de déclaration des cas de cancer (SNDCC) pour la période antérieure à 1992.

Toutes les analyses ont été effectuées par l'Agence de la santé publique du Canada. Les taux d'incidence normalisés selon l'âge (TINA) ont été calculés au moyen de la
Points saillants

- Les tendances liées à l'incidence de certains cancers évoluent rapidement au Canada.

- Les tendances récentes révèlent une augmentation des taux d'incidence de cancer de la glande thyroïde chez les hommes, ce qui incite à étudier les répercussions potentielles de surdiagnostic.

- L'incidence du cancer de la prostate connaît une diminution rapide, ce qui témoigne vraisemblablement des changements récents apportés aux lignes directrices en matière de dépistage.

méthode de normalisation directe en fonction de la répartition par âge de la population canadienne en 2011, pour chaque tranche d'âge de cinq ans. Le logiciel d'analyse Joinpoint ${ }^{4}$ (version 4.6.0.0) a été utilisé pour calculer la variation annuelle en pourcentage (VAP) au moyen des TINA annuels pour chaque type de cancer et pour toute la période (1984-2015), afin de déterminer les années au cours desquelles la VAP avait changé de façon significative. Pour établir la tendance, il fallait disposer d'au moins cinq années de données. Ainsi, la période la plus récente pour établir une tendance était 2011-2015. Par ailleurs, les paramètres par défaut du logiciel Joinpoint ont été utilisés. Au total, 23 types de cancer ont été étudiés.

\section{Résultats et analyse}

Le tableau 1 présente toutes les tendances relevées par Joinpoint entre 1984 et 2015, 
TABLEAU 1

Variation annuelle en pourcentage (VAP) des taux d'incidence normalisés selon l'âge (TINA), selon le siège de cancer et le sexe, Canada (à l'exception du Québec), 1984-2015

\begin{tabular}{|c|c|c|c|c|c|c|c|c|}
\hline \multirow{3}{*}{ Type de cancer } & \multicolumn{4}{|c|}{ Hommes } & \multicolumn{4}{|c|}{ Femmes } \\
\hline & \multicolumn{2}{|c|}{ Année } & \multirow{2}{*}{ VAP } & \multirow{2}{*}{ Valeur $p$} & \multicolumn{2}{|c|}{ Année } & \multirow{2}{*}{ VAP } & \multirow{2}{*}{ Valeur $p$} \\
\hline & de & à & & & de & à & & \\
\hline \multirow[t]{3}{*}{ Bouche } & 1984 & 2004 & $-2,5$ & $<0,001$ & 1984 & 2003 & $-1,0$ & $<0,001$ \\
\hline & 2004 & 2011 & 2,1 & 0,003 & 2003 & 2015 & 0,7 & 0,028 \\
\hline & 2011 & 2015 & $-0,1$ & 0,94 & & & & \\
\hline \multirow[t]{3}{*}{ Csophage } & 1984 & 2006 & 0,3 & 0,020 & 1984 & 2015 & $-0,4$ & $<0,001$ \\
\hline & 2006 & 2010 & 4,3 & 0,068 & & & & \\
\hline & 2010 & 2015 & $-2,4$ & 0,015 & & & & \\
\hline \multirow[t]{2}{*}{ Estomac } & 1984 & 2002 & $-2,5$ & $<0,001$ & 1984 & 1999 & $-3,0$ & $<0,001$ \\
\hline & 2002 & 2015 & $-1,1$ & $<0,001$ & 1999 & 2015 & $-0,8$ & $<0,001$ \\
\hline \multirow[t]{4}{*}{ Colorectal } & 1984 & 1996 & $-0,7$ & $<0,001$ & 1984 & 1996 & $-1,5$ & $<0,001$ \\
\hline & 1996 & 2000 & 0,9 & 0,33 & 1996 & 2000 & 1,2 & 0,23 \\
\hline & 2000 & 2011 & $-0,5$ & 0,001 & 2000 & 2011 & $-0,5$ & $<0,001$ \\
\hline & 2011 & 2015 & $-2,2$ & $<0,001$ & 2011 & 2015 & $-1,9$ & 0,002 \\
\hline \multirow[t]{2}{*}{ Foie } & 1984 & 2011 & 3,8 & $<0,001$ & 1984 & 2015 & 2,7 & $<0,001$ \\
\hline & 2011 & 2015 & 0,2 & 0,88 & & & & \\
\hline \multirow[t]{2}{*}{ Pancréas } & 1984 & 2000 & $-1,5$ & $<0,001$ & 1984 & 2015 & 0,1 & 0,58 \\
\hline & 2000 & 2015 & 0,8 & 0,009 & & & & \\
\hline \multirow[t]{2}{*}{ Larynx } & 1984 & 2015 & $-2,6$ & $<0,001$ & 1984 & 1991 & 0,7 & 0,64 \\
\hline & & & & & 1991 & 2015 & $-3,0$ & $<0,001$ \\
\hline \multirow{4}{*}{$\begin{array}{l}\text { Poumon et } \\
\text { bronches }\end{array}$} & 1984 & 1990 & $-0,6$ & 0,087 & 1984 & 1993 & 2,9 & $<0,001$ \\
\hline & 1990 & 2003 & $-2,2$ & $<0,001$ & 1993 & 2011 & 0,9 & $<0,001$ \\
\hline & 2003 & 2011 & $-0,9$ & 0,002 & & & & \\
\hline & 2011 & 2015 & $-3,3$ & $<0,001$ & 2011 & 2015 & $-1,3$ & 0,043 \\
\hline \multirow[t]{2}{*}{ Mélanome } & 1984 & 2015 & 2,2 & $<0,001$ & 1984 & 1994 & 0,1 & 0,79 \\
\hline & & & & & 1994 & 2015 & 2,0 & $<0,001$ \\
\hline \multirow[t]{2}{*}{ Sein } & 1984 & 2015 & 0,5 & 0,015 & 1984 & 1991 & 2,1 & $<0,001$ \\
\hline & & & & & 1991 & 2015 & $-0,2$ & 0,010 \\
\hline \multirow[t]{3}{*}{ Col de l'utérus } & & & & & 1984 & 2006 & $-2,1$ & $<0,001$ \\
\hline & & & s.o. & & 2006 & 2010 & 1,5 & 0,41 \\
\hline & & & & & 2010 & 2015 & $-3,3$ & $<0,001$ \\
\hline \multirow[t]{4}{*}{ Utérus } & & & & & 1984 & 1990 & $-1,5$ & 0,063 \\
\hline & & & $\mathrm{s}$ & & 1990 & 2006 & 0,5 & 0,013 \\
\hline & & & 3.0. & & 2006 & 2011 & 3,7 & 0,002 \\
\hline & & & & & 2011 & 2015 & 0,1 & 0,92 \\
\hline \multirow[t]{2}{*}{ Ovaire } & & & $\mathcal{S} \Omega$ & & 1984 & 1994 & $-1,7$ & $<0,001$ \\
\hline & & & s.0. & & 1994 & 2015 & $-0,4$ & 0,001 \\
\hline Prostate & 1984 & 1993 & 6,3 & $<0,001$ & & & & \\
\hline & 1993 & 1997 & $-3,0$ & 0,38 & & & & \\
\hline & 1997 & 2001 & 4,1 & 0,23 & & & & \\
\hline & 2001 & 2011 & $-1,6$ & 0,006 & & & & \\
\hline & 2011 & 2015 & $-9,1$ & $<0,001$ & & & & \\
\hline Testicule & 1984 & 2015 & 1,3 & $<0,001$ & & & 0. & \\
\hline Vessie & 1984 & 2009 & $-1,0$ & $<0,001$ & 1984 & 2009 & $-0,9$ & $<0,001$ \\
\hline & 2010 & 2015 & $-1,5$ & 0,052 & 2010 & 2015 & $-1,3$ & 0,18 \\
\hline
\end{tabular}

tandis que la figure 1 détaille la tendance la plus récente. Dans la figure 1 , les données sont présentées pour chaque sexe et sont réparties entre cancers dont l'incidence a connu une hausse ou une baisse significatives (valeur $p<0,05$ ou valeur $p<0,001$ ) et cancers dont l'incidence est demeurée stable (valeur $p \geq 0,05$ ). Nous revenons sur certains de ces résultats dans la suite du texte.

Le taux d'incidence du cancer de la glande thyroïde s'est stabilisé chez les femmes, après avoir augmenté pendant de nombreuses années, mais il connaît toujours une hausse rapide chez les hommes. Cette hausse pourrait être attribuable au surdiagnostic découlant de l'utilisation accrue des technologies de diagnostic comme l'échographie $^{5}$, mais des études récentes montrent également une augmentation de l'incidence des tumeurs papillaires à un stade avancé, suggérant que l'augmentation globale pourrait ne pas être entièrement attribuable au surdiagnostic ${ }^{6}$. L'augmentation de l'incidence du myélome multiple chez les hommes et les femmes pourrait être liée à la prévalence accrue de l'obésitép. Elle pourrait également être attribuable à l'amélioration de la détection et de la vérification des cas, car le taux de myélome est relativement stable dans les pays où la vérification est élevée $e^{8-12}$. Les taux d'incidence du mélanome continuent d'augmenter chez les hommes comme chez les femmes. L'exposition au rayonnement ultraviolet provenant du soleil, des lits de bronzage et des lampes solaires est un facteur de risque bien établi du mélanome ${ }^{13}$. L'augmentation de l'exposition à la lumière ultraviolette en l'absence d'une augmentation correspondante des comportements liés à la protection solaire explique probablement la hausse des taux ${ }^{14}$.

Le cancer du larynx est fortement associé au tabagisme ${ }^{15}$, et la tendance à la baisse observée chez les hommes comme chez les femmes correspond probablement à la tendance à la baisse des taux de tabagisme au Canada $^{16,17}$. Les mêmes observations expliquent probablement la tendance récente du TINA du cancer du poumon chez les hommes et les femmes. Le déclin récent du TINA du cancer colorectal est probablement attribuable en partie à l'augmentation du dépistage, qui permet de détecter des polypes précancéreux traitables. Depuis 2007, la majorité des provinces et des territoires ont mis en œuvre un programme structuré de dépistage du cancer colorec$\operatorname{tal}^{18}$. Bien que cela ne soit pas clairement 
TABLEAU 1 (suite)

Variation annuelle en pourcentage (VAP) des taux d'incidence normalisés selon l'âge (TINA), selon le siège de cancer et le sexe, Canada (à l'exception du Québec), 1984-2015

\begin{tabular}{|c|c|c|c|c|c|c|c|c|}
\hline \multirow{3}{*}{ Type de cancer } & \multicolumn{4}{|c|}{ Hommes } & \multicolumn{4}{|c|}{ Femmes } \\
\hline & \multicolumn{2}{|c|}{ Année } & \multirow{2}{*}{ VAP } & \multirow{2}{*}{ Valeur $p$} & \multicolumn{2}{|c|}{ Année } & \multirow{2}{*}{ VAP } & \multirow{2}{*}{ Valeur $p$} \\
\hline & de & à & & & de & à & & \\
\hline \multirow{4}{*}{$\begin{array}{l}\text { Rein et bassinet du } \\
\text { rein }\end{array}$} & 1984 & 1989 & 4,0 & 0,005 & 1984 & 2015 & 1,0 & $<0,001$ \\
\hline & 1989 & 2003 & 0,1 & 0,64 & & & & \\
\hline & 2003 & 2011 & 2,8 & $<0,001$ & & & & \\
\hline & 2011 & 2015 & $-0,3$ & 0,76 & & & & \\
\hline \multirow[t]{2}{*}{ Encéphale/SNC } & 1984 & 2009 & $-0,2$ & 0,081 & 1984 & 2011 & $-0,3$ & 0,002 \\
\hline & 2009 & 2015 & $-1,9$ & 0,012 & 2011 & 2015 & $-3,2$ & 0,059 \\
\hline \multirow[t]{4}{*}{ Thyroïde } & 1984 & 1997 & 2,8 & 0,002 & 1984 & 1998 & 3,8 & $<0,001$ \\
\hline & 1997 & 2015 & 6,4 & $<0,001$ & 1998 & 2002 & 11,9 & $<0,001$ \\
\hline & & & & & 2002 & 2011 & 6,5 & $<0,001$ \\
\hline & & & & & 2011 & 2015 & 0,1 & 0,94 \\
\hline $\begin{array}{l}\text { Lymphome de } \\
\text { Hodgkin }\end{array}$ & 1984 & 2015 & $-0,4$ & $<0,001$ & 1984 & 2015 & 0,0 & 0,74 \\
\hline \multirow{2}{*}{$\begin{array}{l}\text { Lymphome non } \\
\text { hodgkinien }\end{array}$} & 1984 & 2015 & 1,3 & $<0,001$ & 1984 & 1993 & 2,2 & $<0,001$ \\
\hline & & & & & 1993 & 2015 & 0,9 & $<0,001$ \\
\hline \multirow[t]{2}{*}{ Myélome multiple } & 1984 & 2007 & 0,3 & 0,077 & 1984 & 2015 & 0,6 & $<0,001$ \\
\hline & 2007 & 2015 & 2,6 & $<0,001$ & & & & \\
\hline \multirow[t]{3}{*}{ Leucémie } & 1984 & 1994 & $-0,9$ & 0,067 & 1984 & 2003 & $-0,2$ & 0,18 \\
\hline & 1994 & 2015 & 0,7 & $<0,001$ & 2003 & 2007 & 3,7 & 0,071 \\
\hline & & & & & 2007 & 2015 & $-0,6$ & 0,14 \\
\hline
\end{tabular}

Source des données : Comité consultatif de la Société canadienne du cancer. Statistiques canadiennes sur le cancer 2019. Toronto (Ontario) : Société canadienne du cancer; 2019.

Abréviations : SNC, système nerveux central; s.o., sans objet; VAP, variation annuelle en pourcentage.

indiqué dans le rapport, des taux d'incidence accrus ont été déclarés chez les plus jeunes, ce qui est probablement attribuable en partie à la prévalence de l'obésité19. Le TINA du cancer de l'œsophage est à la baisse tant chez hommes que chez les femmes. Au nombre des facteurs de risque de ce cancer figurent l'obésité, la consommation d'alcool et la consommation de $\operatorname{tabac}^{20}$. Alors que l'obésité ${ }^{7}$ et les ventes de boissons alcoolisées ${ }^{21}$ ont augmenté au Canada, les baisses antérieures de la consommation de $\operatorname{tabac}^{22}$ pourraient expliquer le déclin des taux d'incidence. Le TINA du cancer du foie s'est stabilisé chez les hommes, alors qu'il est toujours à la hausse chez les femmes. Les augmentations liées au type de cancer le plus fréquent, soit le carcinome hépatocellulaire (CHC), sont généralement attribuables aux infections chroniques par le virus de l'hépatite $\mathrm{B}$ et le virus de l'hépatite $C$, ainsi qu'à la consommation excessive d'alcool et au diabète, tous deux en croissance ${ }^{23}$. La prévalence du CHC étant plus élevée dans certains pays à faible revenu, l'augmentation au
Canada pourrait s'expliquer en partie par l'augmentation du nombre d'immigrants provenant de régions où le $\mathrm{CHC}$ est fréquent, en particulier certaines régions de l'Asie et de l'Afrique ${ }^{24}$.

Le TINA du cancer du sein chez la femme décline lentement depuis 1991. Cette tendance est vraisemblablement attribuable au dépistage mammographique et aux modifications à long terme des facteurs de risque $^{25}$. Le cancer du col de l'utérus connaît une baisse, explicable en grande partie par le dépistage systématique au moyen du test de Papanicolaou (Pap). Toutes les provinces du Canada (à l'exception du Québec) ont mis en place un programme organisé de dépistage du cancer du col de l'utérus. Il est recommandé dans les lignes directrices actuelles de procéder au dépistage tous les deux ou trois ans, à partir de 21 ou 25 ans et jusqu'à 65 ou $70 \mathrm{ans}^{26}$. Au cours des prochaines années, le taux d'incidence du cancer du col de l'utérus devrait continuer à diminuer grâce à la vaccination contre le virus du papillome humain $^{27}$. Au fil du temps, le TINA du cancer de la prostate a suivi la même tendance que l'utilisation du dépistage au moyen du dosage de l'antigène prostatique spécifique (APS) au Canada ${ }^{28}$. En 2014, le Groupe d'étude canadien sur les soins de santé préventifs a déconseillé le dépistage par dosage de l'APS chez les hommes de tous âges en raison du manque de données probantes sur les avantages ainsi qu'en raison des risques de surdiagnostic et des inconvénients liés à un traitement non nécessaire $^{29}$. À l'heure actuelle, on observe une diminution rapide des TINA du cancer de la prostate.

En 2014, Action Cancer Ontario a mis en œuvre un nouveau système de déclaration des cas de cancer, qui s'est traduit par plusieurs améliorations au chapitre du recensement des cas de cancer, notamment l'enregistrement des cas de cancer de la vessie in situ, qui n'avaient pas été enregistrés auparavant ${ }^{30}$. Les données recueillies depuis 2010 ont été ajoutées au système de façon rétrospective, ce qui a donné lieu à une augmentation apparente des cas nouveaux de cancer de la vessie à partir de 2010. Bien que la tendance à la baisse du taux d'incidence du cancer de la vessie entre 2010 et 2015 soit comparable à celle observée entre 1984 et 2009, cette période est trop courte pour que l'observation soit significative. Nous avons eu à créer une analyse Joinpoint en 2010 pour rendre compte de ces données rétrospectives.

Les cancers de l'encéphale et du système nerveux central (SNC) ont connu une baisse annuelle de 3,9 \% chez les femmes entre 2011 et 2015, mais non statistiquement significative ( $p=0,059)$. Cette absence de signification statistique s'explique probablement par la courte période visée et par la variabilité des taux annuels. Chez les hommes, on assiste à une baisse significative de 1,9 \% par année depuis 2009 .

\section{Conclusion}

D’après nos résultats, les tendances en matière d'incidence du cancer au Canada sont dynamiques et spécifiques à chaque type de cancer. Les dernières tendances révèlent une augmentation des taux de cancer de la glande thyroïde chez les hommes, ce qui incite à vérifier les répercussions potentielles de surdiagnostic sur l'incidence du cancer. À l'inverse, les taux d'incidence des autres cancers ont récemment diminué, plus particulièrement pour 
FIGURE 1

Variation annuelle en pourcentage (VAP) la plus récente ${ }^{\mathrm{a}}$ des taux d'incidence normalisés selon l'âge (TINA), selon le siège de cancer et le sexe, Canada (à l'exception du Québec)

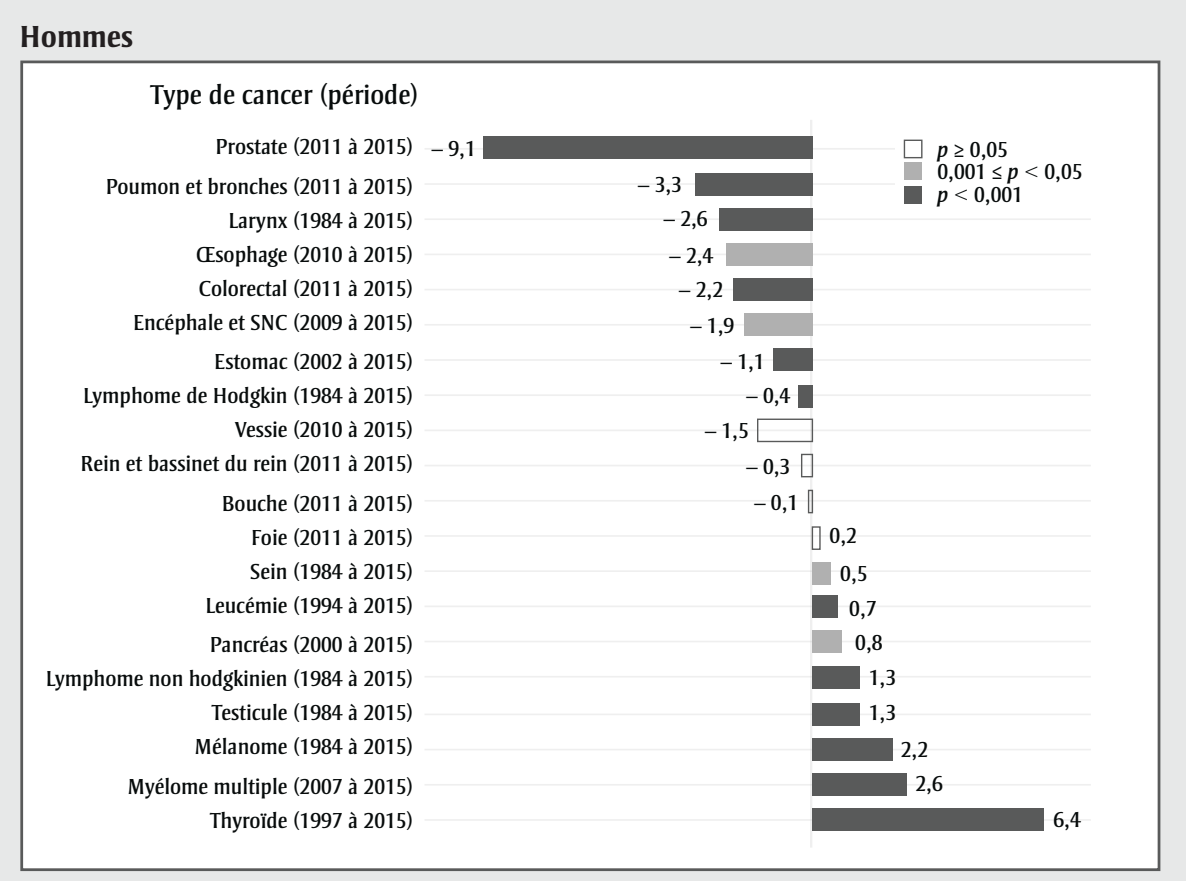

Femmes

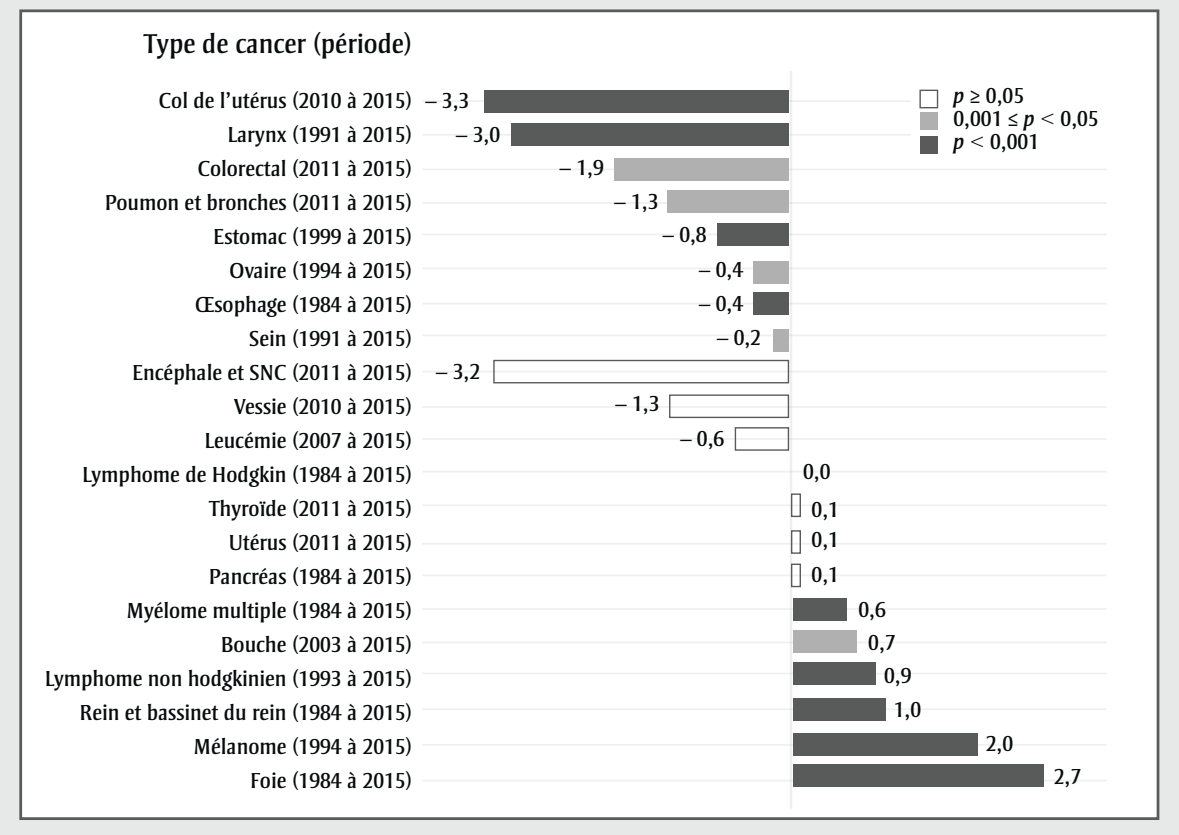

Source des données : Comité consultatif de la Société canadienne du cancer. Statistiques canadiennes sur le cancer 2019. Toronto (Ont.) : Société canadienne du cancer; 2019.

Abréviation : SNC, système nerveux central.

a Les VAP ont été calculées pour la période 1984 à 2015. Si un ou plusieurs changements significatifs ont été observés dans la tendance des taux, la VAP reflète la tendance depuis le plus récent changement significatif jusqu'en 2015. Si aucun changement significatif n'a été observé, la VAP reflète la tendance des taux sur toute la période.

le cancer de la prostate et celui du col de l'utérus. Le déclin des taux d'incidence de ces deux cancers met en évidence les répercussions potentielles de l'amélioration des lignes directrices en matière de dépistage fondées sur les données probantes. Plus précisément, la diminution du surdépistage (cancer de la prostate) et la mise en œuvre d'un dépistage systématique (cancer du col de l'utérus) peuvent avoir mené à un déclin de l'incidence.

\section{Conflits d'intérêts}

Les auteurs déclarent n’avoir aucun conflit d'intérêts.

\section{Contributions des auteurs et avis}

Tous les auteurs ont contribué à la conception, à la conceptualisation et à la révision du manuscrit. La mise en place ainsi que l'analyse et l'interprétation des données ont été réalisées par l'Agence de la santé publique du Canada.

Le contenu de l'article et les points de vue qui y sont exprimés n'engagent que les auteurs; ils ne correspondent pas obligatoirement à ceux du gouvernement du Canada.

\section{Références}

1. Gouvernement du Canada. Le diabète au Canada [Internet]. Ottawa (Ont.) : Gouvernement du Canada; 2017 [modification le 14 novembre 2017; consultation le 3 juillet 2019]. En ligne à : https://www.canada.ca/fr/sante -publique/services/publications/maladies -et-affections/diabete-canada-faits -saillants-systeme-surveillance-maladies -chroniques.html

2. Comité consultatif des statistiques canadiennes sur le cancer. Statistiques canadiennes sur le cancer 2019. Toronto (Ontario) : Société canadienne du cancer; 2019. 98 p.

3. Statistique Canada. Registre canadien du cancer (RCC) [Internet]. Ottawa (Ontario) : Gouvernement du Canada; 2019 [modification le 28 janvier 2019; consultation le 5 mars 2019]. En ligne à : http://www23.statcan.gc.ca/imdb /p2SV_f.pl?Function = getSurvey $\&$ SDDS $=3207$

4. National Cancer Institute, Division of Cancer Control \& Population Sciences, Surveillance Research Program. Joinpoint trend analysis software, version 4.6.0.0. Bethesda (MD): National Cancer Institute; 2019 [consultation le 6 mars 2019]. En ligne à : https://surveillance .cancer.gov/joinpoint 
5. Vaccarella S, Dal Maso L, Laversanne $\mathrm{M}$, et al. The impact of diagnostic changes on the rise in thyroid cancer incidence: a population-based study in selected high-resource countries. Thyroid. 2015;25(10):1127-1136.

6. Lim H, Devesa SS, Sosa JA, Check D, Kitahara CM. Trends in thyroid cancer incidence and mortality in the United States, 1974-2013. JAMA. 2017;317(13): 1338-1348.

7. Twells LK, Gregory DM, Reddigan J, et al. Current and predicted prevalence of obesity in Canada: a trend analysis. CMAJ Open [Internet]. 2014;2(1):E18E26. En ligne à : http://cmajopen.ca /content/2/1/E18.long

8. Velez R, Turesson I, Landgren $\mathrm{O}$, Kristinsson SY, Cuzick J. Incidence of multiple myeloma in Great Britain, Sweden, and Malmo, Sweden: the impact of differences in case ascertainment on observed incidence trends. BMJ Open [Internet]. 2016;6(1):e009584.

9. Andres M, Feller A, Arndt V, NICER Working Group. Trends of incidence, mortality, and survival of multiple myeloma in Switzerland between 1994 and 2013. Cancer Epidemiol. 2018;53: 105-110.

10. Kyle RA, Therneau TM, Rajkumar SV, Larson DR, Plevak MF, Melton LJ 3rd. Incidence of multiple myeloma in Olmsted County, Minnesota: trend over 6 decades. Cancer. 2004;101(11): 2667-2674.

11. Renshaw C, Ketley N, Moller H, Davies EA. Trends in the incidence and survival of multiple myeloma in South East England 1985-2004. BMC Cancer. 2010;10:74.

12. Soutar RL, Dawson AA, Wilson BJ. Multiple myeloma in north east Scotland: a review of incidence and survival over three decades. Health Bull. 1996;54(3):232-240.

13. Centre international de recherche sur le cancer. Exposure to artificial UV radiation and skin cancer. Genève : Organisation mondiale pour la santé; 2006. 76 p.
14. National Skin Cancer Prevention Committee. Exposure to and protection from the sun in Canada: a report based on the 2006 Second National Sun Survey. Toronto (Ont.): Canadian Partnership Against Cancer; 2010.

15. IARC Working Group on the Evaluation of Carcinogenic Risks to Humans. Tobacco smoke and involuntary smoking. IARC Monogr Eval Carcinog Risks Hum. 2004;83:1-1438.

16. Statistique Canada. Enquête canadienne sur le tabac, l'alcool et les drogues (ECTAD) : sommaire de 2013 [Internet]. Ottawa (Ont.) : Santé Canada; 2015 [modification le 3 février 2015; consultation le 30 mars 2019]. En ligne à : https://www.canada.ca/fr/sante -canada/services/enquete-canadienne -tabac-alcool-et-drogues/sommaire -2013.html

17. Santé Canada. Enquête de surveillance canadienne de la consommation d'alcool et de drogues (ESCCAD) [Internet]. 2014 [modification le 8 avril 2014; consultation le 30 mars 2019]. En ligne à : https://www.canada.ca/fr /sante-canada/services/preoccupations -liees-sante/prevention-traitement -toxicomanie/enquete-surveillance -canadienne-consommation-alcool -drogues.html

18. Major D, Bryant H, Delaney M, et al Colorectal cancer screening in Canada: results from the first round of screening for five provincial programs. Curr Oncol 2013;20(5):252-7.

19. Cancer Care Ontario. Cancer fact Colorectal cancer incidence increasing in younger adults [Internet]. 2016 [consultation le 30 mars 2019]. En ligne à : https://www.cancercareontario.ca /en/cancer-facts/colorectal-cancer -increasing-younger-adults

20. Otterstatter MC, Brierley JD, De P, et al. Esophageal cancer in Canada: trends according to morphology and anatomical location. Can J Gastroenterol. 2012; 26(10):723-727.

21. Agriculture et Agroalimentaire Canada. Tendances de la consommation : vin, bière et spiritueux au Canada. Ottawa (Ont.) : Gouvernement du Canada; 2013. $21 \mathrm{p}$
22. Organisation for Economic Co-operation Development (OECD). Daily smokers. 2018 [consultation le 30 mars 2019]. En ligne à : https://data.oecd.org /healthrisk/daily-smokers.htm

23. Trad D, Bibani N, Sabbah M, et al. Known, new and emerging risk factors of hepatocellular carcinoma (review). Presse Med 2017;46(11):1000-1007.

24. Bosetti C, Turati F, La Vecchia C. Hepatocellular carcinoma epidemiology. Best Pract Res Clin Gastroenterol. 2014;28(5):753-770.

25. Holford TR, Cronin KA, Mariotto AB, et al. Changing patterns in breast cancer incidence trends. J Natl Cancer Inst Monogr. 2006;(36):19-25.

26. Partenariat canadien contre le cancer. Dépistage du cancer du col de l'utérus au Canada : analyse de l'environnement (2018). Toronto (Ont.) : Partenariat canadien contre le cancer; 2018 [consultation le 30 mars 2019]. En ligne à : https://www.partnershipagainst cancer.ca/fr/topics/cervical-cancer -screening-environmental-scan-2018

27. Comité consultatif de la Société canadienne du cancer. Statistiques canadiennes sur le cancer 2016. Toronto (Ont.) : Société canadienne du cancer; 2016. 152 p.

28. LeBlanc AG, Demers A, Shaw A. Tendances récentes liées au cancer de la prostate au Canada. Rapports sur la santé. 2019;30(4):14-20.

29. Bell N, Connor Gorber S, Shane A, et al. Recommendations on screening for prostate cancer with the prostate-specific antigen test. CMAJ 2014;186(16):12251234.

30. Cancer Care Ontario. Ontario cancer statistics 2018. Toronto (Ont.): Cancer Care Ontario; 2018. 172 p 